\title{
Amiodarone in congestive heart failure: unravelling the GESICA and CHF-STAT differences
}

Patients with congestive heart failure are at an increased risk of sudden death. Heart failure is the most common cardiovascular discharge diagnosis in elderly patients in the United States, and its incidence more than doubles each decade after the age of 45 . The aging population will further increase the number of patients in whom congestive heart failure is diagnosed. Two recent studies have evaluated the role of amiodarone in improving survival in patients with congestive heart failure. The Survival Trial Of Antiarrhythmic Therapy In Congestive Heart Failure (CHF-STAT) ${ }^{1}$ was performed in Veterans' Administration hospitals in the United States, and the Gruppo de Estudo de la Sobrevida en la Insuficiencia Cardiaca en Argentina (GESICA) was performed in Argentina. ${ }^{2}$ Unfortunately, the studies had divergent results, making clear recommendations difficult.

Arrhythmia in patients with congestive heart failure Recent studies using angiotensin converting enzyme (ACE) inhibitors have demonstrated significantly increased survival in patients with moderately symptomatic congestive heart failure. In the SOLVED and V-HeFT II trials, the 2.5 year mortality in patients treated with ACE inhibitors was $25-27 \%$ with $23 \%$ of patients in SOLVED dying an arrhythmic death without worsening congestive heart failure, and $57 \%$ of patients in V-HeFT II dying suddenly with or without warning symptoms. Thus about $25-50 \%$ of deaths in patients with congestive heart failure are related to arrhythmia (both tachyarrhythmia and bradyarrhythmia). Amiodarone was chosen in GESICA and CHF-STAT because of its low risk of proarrhythmia ${ }^{3}$ and of worsening heart failure in patients with already reduced ventricular function. Previous studies of amiodarone in patients with cardiomyopathy, ventricular arrhythmias, and congestive heart failure have reported inconsistent results: Neri et $a^{4}$ reported an improved survival, Cleland et $a l^{5}$ reported a decreased incidence of sudden death, and Nicklas $e t a l^{6}$ reported no improvement.

\section{GESICA}

This trial openly randomised 516 patients optimally treated for congestive heart failure to receive either amiodarone $(600 \mathrm{mg}$ daily for 14 days followed by $300 \mathrm{mg}$

\footnotetext{
Glossary

CHF-STAT = survival trial of antiarrhythmic therapy in congestive hear failure

GESICA = gruppo de estudio de la sobrevida en la insuficiencia cardiaca en Argentina

SOLVED = studies of left ventricular dysfunction

$\mathrm{V}$-HeFT = Veterans heart failure trial

CAMIAT = Canadian amiodarone myocardial infarction arrhythmia trial

CASCADE = conventional versus amiodarone drug evaluation trial

BASIS = basal antiarrhythmic study of infarct survival
}

daily) or no additional treatment. The mean aged was 59 years and $80 \%$ were male. Eighty per cent of patients had class III or IV heart failure with associated myocardial infarction in $40 \%$, idiopathic dilated cardiomyopathy in $20 \%$, alcoholism in $30 \%$, and Chagas' disease in $10 \%$. Patients with angina were excluded. Mean follow up was 13 months with $33.5 \%$ deaths in the amiodarone group and $41.4 \%$ deaths in the control group for a risk reduction of $28 \%(P=0.024)$. In the amiodarone group, there was a $27 \%(P=0.16)$ reduction in sudden death, a $23 \%$ $(P=0.16)$ reduction in progressive congestive heart failure, and a $31 \%(P=0.002)$ reduction in hospital admissions for worsening congestive heart failure. The reduction in mortality and hospital admission was present in subgroup analysis for gender, heart failure class, and presence or absence of nonsustained ventricular tachycardia. Two year survival in the amiodarone group was approximately $60 \%$ compared with $45 \%$ in the control group. Five per cent of patients stopped amiodarone because of side effects ( $0.35 \%$ per month of follow up). The authors concluded that low dose amiodarone was an effective and reliable treatment that reduced both mortality and hospital admissions in patients with severe congestive heart failure, and these benefits were independent of the presence of complex ventricular arrhythmias.

\section{CHF-STAT}

This recently published trial blindly randomised 674 patients optimally treated for congestive heart failure to receive either amiodarone $(800 \mathrm{mg}$ daily for 14 days, then $400 \mathrm{mg}$ daily for 50 weeks, then $300 \mathrm{mg}$ daily) or placebo in addition to their conventional treatment. The mean age was 65 years and $99 \%$ were male. Forty two per cent of patients had class III or IV heart failure which was due to coronary artery disease in $\mathbf{7 2 \%}$ and dilated cardiomyopathy in $28 \%$. Mean follow up was 45 months with no significant survival difference between the two treatment groups $(P=0.6)$. The 2 year actuarial survival rate was $69 \cdot 4 \%$ for patients in the amiodarone group and $70.8 \%$ for patients in the placebo group. There was a trend toward a reduction in overall mortality among patients with nonischaemic cardiomyopathy who received amiodarone $(P=0.07)$. Amiodarone did improve left ventricular ejection fraction by $42 \%$ at 2 years. Twenty seven per cent of patients stopped amiodarone because of side effects $(0.6 \%$ per month of follow up). This trial showed that amiodarone was effective in suppressing ventricular arrhythmias and improving ventricular function but did not reduce the incidence of sudden death or prolong survival in patients with congestive heart failure. There was a trend toward reduced mortality among patients with a nonischaemic cardiomyopathy.

Why did the two trials reach different conclusions? There is no clear and easy explanation for the different results in the two trials. GESICA supports the routine use 
of amiodarone in patients with congestive heart failure while CHF-STAT does not. Differences between the two trials include the patient's age, the predominance of men in CHF-STAT, severity of heart failure, adherence to treatment, and difference in underlying heart disease.

CHF-STAT patients were approximately 6 years older which should have had no important influence on the outcome. Ninety nine per cent of CHF-STAT patients were male compared with $79 \%$ of GESICA patients. Increased body surface area in males may have decreased the relative efficacy of amiodarone (both studies used a maintenance dose of $300 \mathrm{mg}$ a day). However, this should have less effect because of the increased dosing regimen for CHFSTAT which resulted in a first-year cumulative dose of amiodarone that was $33 \%$ higher. Other studies of amiodarone have not reported a difference in efficacy based on gender. ${ }^{78}$ The GESICA patients had more advanced congestive heart failure with nearly $80 \%$ in class III or IV compared with $42 \%$ in CHF-STAT. In addition, 2 year mortality in the control group of GESICA was $55 \%$ compared with $30 \%$ in CHF-STAT. It is possible that patients who have advanced heart failure with an increased risk of mortality will show a more striking benefit from a medicine that is only marginally effective in a healthier population. This argument is less persuasive in the context of the BASIS trial ${ }^{7}$ where amiodarone efficacy in patients after myocardial infarction was only significant in those with an ejection fraction greater than $40 \%$.

More patients in CHF-STAT than in GESICA stopped taking amiodarone because of side effects. The $27 \%$ of patients who stopped amiodarone during the mean follow up of 45 months resembles other studies such as CAS$\mathrm{CADE}^{9}$ where $25 \%$ of patients stopped amiodarone at 2 years, and the CAMIAT ${ }^{10}$ pilot study where $35 \%$ of patients discontinued amiodarone at 20 months. This rate of drug discontinuation should not have accounted for the divergent results in the studies.

The most striking difference between the two studies is the nature of the underlying heart disease. In CHF-STAT, $72 \%$ of patients had ischaemic cardiomyopathy compared with only $40 \%$ of patients with a history of myocardial infarction in GESICA. Patients with any angina within 3 months of entry were excluded. The remaining patients in GESICA had either idiopathic cardiomyopathy or cardiomyopathy secondary to alcoholism or Chagas' disease. Although previous studies ${ }^{78}$ showed that amiodarone is effective in preventing subsequent death in patients after acute myocardial infarction, BASIS, ${ }^{7}$ as previously mentioned, showed that this effect was not present in patients with ejection fraction $<40 \%$. Patients in CHF-STAT with ischaemic cardiomyopathy may have had a similar lack of benefit from amiodarone. Such patients may also be liable to bradyarrhythmias related to advancing ischaemia which would be aggravated by amiodarone, resulting in death from bradycardia. The $30 \%$ of patients who were alcoholic and $10 \%$ who had Chagas' disease in GESICA may be patients who show a unique response to amiodarone. CHF-STAT did show a trend toward mortality reduction among patients with non-ischaemic cardiomyopathy who received amiodarone, a finding similar to GESICA.

\section{Sudden cardiac death in heart failure: trial of prophylactic amiodarone versus implantable defibrillator therapy (SCD-HeFT)}

SCD-HeFT was prompted by the failure of previous trials to come to consistent and helpful conclusions. This trial compares conventional treatment alone versus addition of amiodarone versus addition of a pectorally implanted third generation implantable cardioverter defibrillator in 2500 patients with an ejection fraction $\leqslant 35 \%$, class II or III congestive heart failure, and idiopathic or ischaemic cardiomyopathy. The addition of a defibrillator capable of bradycardia pacing will presumably prevent death from both tachyarrhythmia and bradyarrhythmia. The pilot study enrolment has been completed. This study will be put in context with GESICA and CHF-STAT to help define optimal treatment to prevent arrhythmic deaths in patients with congestive heart failure.

\section{Clinical implications and recommendations}

Clear enthusiastic recommendations concerning the approach to prevention of arrhythmic death in patients with congestive heart failure cannot come from these two conflicting studies. The studies have demonstrated the general safety of amiodarone with a low risk of proarrhythmia in such patients. Although side effects were present, they were not deemed life threatening and did not seem to increase mortality. The drug is safe in this group of patients and improves ventricular function (as observed in CHF-STAT) and decreases hospital admissions because of progressive congestive heart failure (as observed in GESICA). Based on these findings, amiodarone is the preferred drug to treat supraventricular arrhythmias in patients with congestive heart failure. The drug is effective in suppressing ventricular arrhythmias and therefore should be the drug of choice in patients with symptomatic ventricular arrhythmias associated with reduced ventricular function who require an antiarrhythmic drug. Both studies suggest that amiodarone may improve survival in patients with non-ischaemic heart disease and should be considered in such patients. Amiodarone cannot be recommended for use in patients with ischaemic cardiomyopathy and asymptomatic ventricular arrhythmias.

Mayo Clinic, STEPHEN C HAMMILI DOUGLAS L PACKER

200 First Street Southwest,

Rochester,

Minnesota 55905

USA

1 Singh SN, Fletcher RD, Fisher SG, et al. Amiodarone in patients with congestive heart failure and asymptomatic ventricular arrhythmia. $N$ Engl f Med 1995;333:77-82.

2 Doval HC, Nul DR, Grancelli HO, et al. Randomized trial of low-dose amiodarone in severe congestive heart failure. Lancet 1994;344:493-8.

3 Hohnloser SH, Klingenheben T, Singh BN. Amiodarone associated proarrhythmic effects. Ann Intern Med 1994;121:529-35.

4 Neri R, Mestroni L, Salvi A, Pandullo C, Camerini F. Ventricular arrhythmias in dilated cardiomyopathy: efficacy of amiodarone. Am Heart 1987;113:707-15.

5 Cleland JG, Dargie HJ, Ford I. Mortality in heart failure: clinical variables of prognostic value. $B r$ Heart $f$ 1987;58:572-82.

6 Nicklas JM, McKenna WJ, Stewart RA, et al. Prospective, double-blind, placebo controlled trial of low-dose amiodarone in patients with severe heart failure and asymptomatic frequent ventricular ectopy. Am Heart $\mathcal{F}$ 1991;122:1016-21.

7 Burkart F, Pfeisterer M, Kiowksi W, Follath F, Burckhardt D. Effect of antiarrhythmic therapy on mortality in survivors of myocardial infarction with asymptomatic complex ventricular arrhythmias: Basal antiarrhyth mic study of infarct survival (BASIS). 7 Am Coll Cardiol 1990;16:1711-8.

Ceremuzynski L, Kleczar E, Krzeminska-Pakula M, et al. Effect of amiodarone on mortality after myocardial infarction: a double blind, placebo darone on mortality after myocardial infarction: a double

9 CASCADE Investigators. Randomized antiarrhythmic drug therapy in survivors of cardiac arrest. $A m \mathcal{F}$ Cardiol 1993;72:280-7.

10 Carins JA, Connolly SJ, Gent M, Roberts R. Post myocardial infarction mortality in patients with ventricular premature depolarizations. Canadian amiodarone myocardial infarction arrhythmia trial pilot study. Circulation 1991;84:550-7. 